\title{
Observación de capas de grafeno mediante contraste óptico y dispersión Raman*
}

\author{
Claudia Bautista Flores, ${ }^{1}$ Roberto Ysacc Sato Berrú, ${ }^{2}$ \\ Doroteo Mendoza López ${ }^{3}$
}

\begin{abstract}
Resumen: En este trabajo hemos usado la técnica de contraste óptico como herramienta de identificación preliminar y sencilla de capas de grafeno, la cual es más simple en comparación con la propuesta de otros autores y es validada con espectroscopia Raman. Para ello obtenemos grafeno y pocas capas de grafeno por exfoliación mecánica de grafito Kish. Adquirimos imágenes digitales, a través de un microscopio óptico, de las diferentes muestras depositadas sobre un sustrato de silicio con una película de óxido de silicio de $306.2 \mathrm{~nm}$. Con un programa de análisis de imágenes adquirimos la componente verde de los números RGB, tanto de la muestra como del sustrato; con ello, calculamos el contraste óptico y determinamos el número de capas de grafeno. Logramos determinar de una a cuatro capas de grafeno.

Palabras Clave: Contraste óptico, capas de grafeno, electroscopía Raman.
\end{abstract}

ABSTRACT: We used the optical contrast technique to identify layers of graphene, in a simpler way in contrast with other authors; this technique was validated by Raman spectroscopy. Layers of graphene were obtained by mechanical exfoliation of Kish graphite, and were deposited on silicon substrate with a $306.2 \mathrm{~nm}$ film of silicon oxide. Digital images of these samples were taken to use green component of the RGB numbers in the optical contrast calculation. We can identify from one to four layers of graphene.

KEY WORDS: Optical contrast, layers of graphene, Raman spectroscopy.

\section{INTRODUCCIÓN}

El grafeno es un material bidimensional de átomos de carbono, una red tipo panal de abeja (figura 1a). Debido a que es un material bidimensional, se dudaba que pudiera existir en la naturaleza aislado del grafito en bulto (Landau, 1969). En 2004, Novoselov y Geim lograron separarlo del grafito usando exfoliación mecánica con cinta adhesiva (Novoselov et al., 2004). La separación del grafeno del grafito y la serie de investigaciones que este equipo realizó, les valió la obtención del premio Nobel de Física en el 2010. Este material ha probado tener interesantes propiedades. Es el material más delgado que se ha encontrado, con un grosor de tan sólo $0.34 \mathrm{~nm}$, un semiconductor sin brecha prohibida de energía y altamente conductivo (Novoselov et al., 2005). Los electrones en el material pueden viajar distancias submicrométri-

\footnotetext{
* Agradecemos el apoyo en la elaboración de este artículo al Laboratorio de Nanomateriales del CCADETUNAM.

1 Instituto de Investigaciones en Materiales (IIM), UNAM, Apartado postal 70-360, 04510 México, D. F. (claudiabautistaf@gmail.com), Tel. 55484549 21, Fax: 56161251.

2 Centro de Ciencias Aplicadas y Desarrollo Tecnológico, Circuito Exterior s/n, UNAM, Apdo. Postal 70-186, 04510, México, D. F. (roberto.sato@ccadet.unam.mx).

3 IIM-UNAM, (doroteo@unam.mx).
} 
cas sin dispersarse (Geim, 2008). Presenta un efecto Hall cuántico fraccional (Yuanbo et al., 2005) y la paradoja de Klein también se ha observado experimentalmente en el grafeno (Stander et al., 2009). El grafeno es estructuralmente maleable, pero el esfuerzo y las deformaciones producen cambios en sus propiedades ópticas, electrónicas y fonónicas (Pereira et al., 2008). Sólo absorbe el 2.3\% de la luz, mientras que el 97.7\% es transmitida (Nair et al., 2008; Flores y Mendoza, 2011). En multicapas de grafeno se ha modulado la transmitancia óptica mediante una señal eléctrica (Benítez y Mendoza, 2013). Posee una conductividad mínima de $4 e^{2} / h$ (Novoselov et al., 2005; Nair et al., 2008) puede ser deformado más de un 15 \% (Pereira et al., 2008; Lee et al., 2008), y es impermeable a gases (Bunch et al., 2008). Debido a sus peculiares propiedades (Flores et al., 2012), puede aplicarse principalmente en el área de la electrónica. Se han fabricado transistores de efecto de campo que operan a $100 \mathrm{GHz}$ (Lin et al., 2010) y sensores de tamaño micrométrico (Schedin et al., 2007). Se puede usar en capacitores (Liu et al., 2010), celdas solares (Wang et al., 2008) y puntos cuánticos (Wang et al., 2010; Geim et al., 2007; Moriyama et al., 2010; Ponomarenko et al., 2008). En este trabajo presentamos la forma de producir grafeno por exfoliación mecánica partiendo de cristales de grafito y un método óptico para identificar el número de capas de grafeno, el cual se emplea de manera rutinaria antes de usar espectroscopia Raman (Yan et al., 2007; Blake et al., 2008, Alexeev et al., 2013), por ello puede ser implementado en un laboratorio escolar que cuente con un microscopio óptico en el modo de reflexión y una cámara digital. Este método se compara y respalda satisfactoriamente empleando dispersión Raman, como un método alternativo para caracterizar al grafeno (Ferrari et al., 2006).

\section{FuNDAMENTOS TEÓRICOS DEL CONTRASTE ÓPTICO}

El grafeno, depositado en un sustrato de silicio con óxido de silicio, se observó por primera vez en un microscopio óptico. Una técnica cuantitativa para determinar si lo que vemos al microscopio óptico es o no grafeno es la del contraste óptico (Blake et al., 2007), el cual se define como:

$$
C=\frac{I_{S}-I_{G}}{I_{S}}
$$

En donde $I_{S}$ es la intensidad de la luz reflejada por el sustrato, mientras que $I_{G}$ la reflejada por las capas de grafeno sobre el sustrato. En la figura $1 \mathrm{~b}$ se muestra un esquema del grafeno sobre el sustrato de silicio con óxido de silicio. En esta misma figura se observan rayos de luz que inciden en el sistema y otros que son reflejados por los diferentes materiales. $n_{0}, n_{1}, n_{2}$ y $n_{3}$ son los índices de refracción de cada material; $d_{1}$ es el grosor del o las capas de grafeno y $d_{2}$ el grosor del óxido de silicio.

Usando teoría de Fresnel, podemos calcular la intensidad de la luz reflejada por un sistema de 2 capas (grafeno y óxido de silicio) sobre un sustrato seminfinito (silicio), como el del esquema de la fig. 1b, tal intensidad tiene la siguiente forma (Anders, 1972):

$$
I_{G}=\left|r^{(2)} e^{i \varepsilon^{(2)}}\right|^{2}=\left|\frac{r_{1}+r_{2} e^{-i \Delta_{1}}+r_{3} e^{-i\left(\Delta_{1}+\Delta_{2}\right)}+r_{1} r_{2} r_{3} e^{-i \Delta_{2}}}{1+r_{1} r_{2} e^{-i \Delta_{1}}+r_{1} r_{3} e^{-i\left(\Delta_{1}+\Delta_{2}\right)}+r_{2} r_{3} e^{-i \Delta_{2}}}\right|^{2}
$$



forma:

Como la luz incide normalmente, los coeficientes de Fresnel tienen la siguiente

$$
r_{1}=\frac{n_{0}-n_{1}}{n_{0}+n_{1}}, \quad r_{2}=\frac{n_{1}-n_{2}}{n_{1}+n_{2}} \quad \text { y } \quad r_{3}=\frac{n_{2}-n_{3}}{n_{2}+n_{3}}
$$

Y los corrimientos de fases en el camino óptico dentro de la película son:

$$
\Delta_{1}=\frac{2 \pi n_{1} d_{1}}{\lambda} \text { y } \Delta_{2}=\frac{2 \pi n_{2} d_{2}}{\lambda}
$$

Para la intensidad de la luz reflejada por el sustrato $I_{S^{\prime}}$ en (1) simplemente hacemos $n_{1}=1$, lo cual indica que el sistema sólo consiste de una capa de óxido de silicio sobre el sustrato infinito de silicio. Usamos $n_{0}=1$ como el índice de refracción del aire y $n_{1}=2.3-i 1.6$ para el grafeno (Palik, 1991). Los valores de $n_{2}$ y $n_{3}$ dependen de la longitud de onda (Palik, 1991), así que se deben ajustar al polinomio que mejor se ajuste y sustituirlos donde correspondan en (3) y (4). En nuestro caso usamos $n_{2}=1.4601 \mathrm{y}$ $n_{3}=4.097-i 0.044$, cuando la longitud de onda usada fue de $546.1 \mathrm{~nm}$.

FIGURA 1. a) Red tipo panal de abeja de átomos de carbono; b) Esquema de grafeno sobre un sustrato de silicio con óxido de silicio. $I_{S}$ es la intensidad de la luz reflejada por el sustrato formado por una capa de $\mathrm{SiO}_{2}$ sobre un sustrato seminfinito de $\mathrm{Si}$. $\mathrm{I}_{\mathrm{C}}$ es la intensidad de la luz reflejada por grafeno, con un grosor de $d_{1}=0.34 \mathrm{~nm}$, sobre el sustrato descrito anteriormente; c) contraste óptico calculado para el esquema en b), los números en las gráficas corresponden al número de capas de grafeno; d) contraste óptico contra grosor de óxido de silicio, correspondiente a una sola capa de grafeno y una longitud de onda incidente de $546.1 \mathrm{~nm}$. En la figura se señala el contraste para el caso de $306.2 \mathrm{~nm}$ de óxido de silicio.
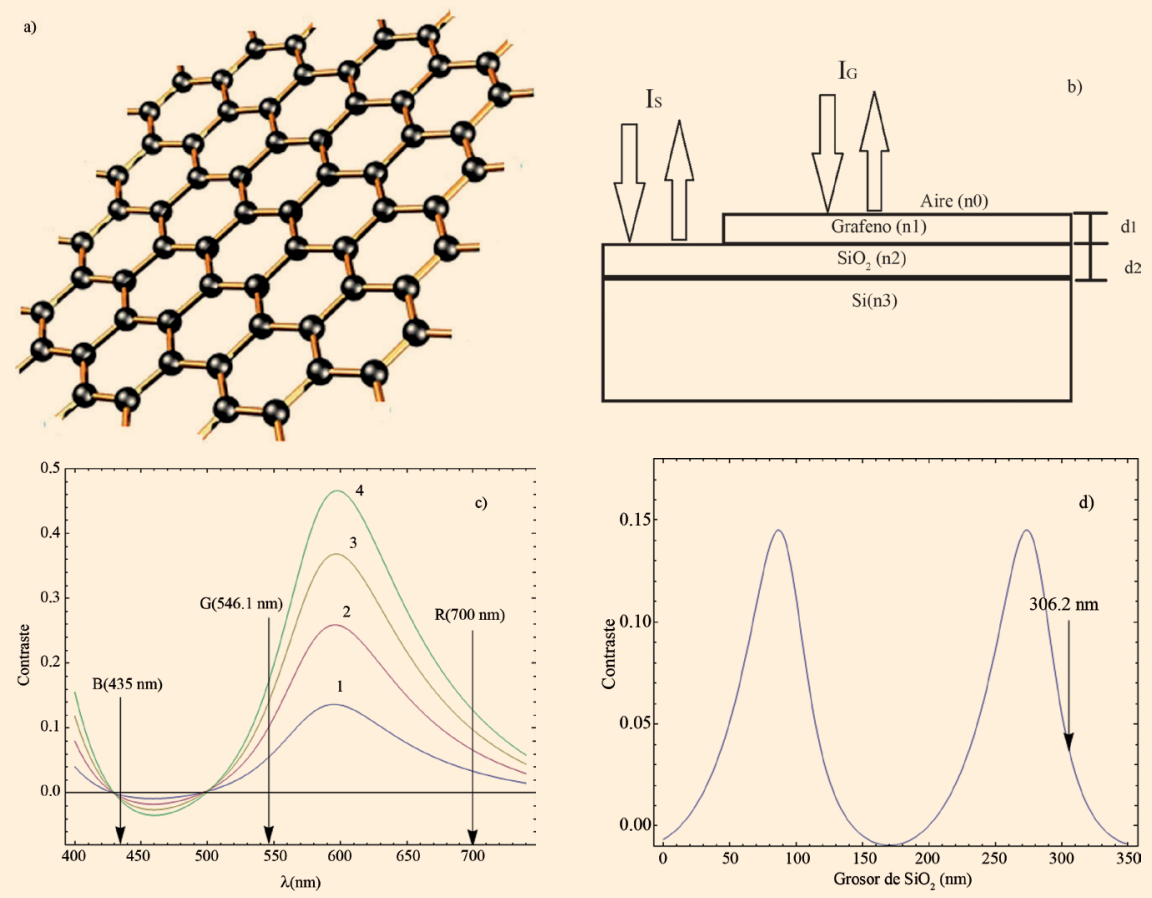
Sustituyendo $I_{G}$ y $I_{S}$ para un grosor de $306.2 \mathrm{~nm}$ (medido con elipsometria) para el $\mathrm{SiO}_{2}$, se encuentra que el contraste tiene la forma de la fig. 1c; donde se ha usado que el grosor del grafeno es de $0.34 \mathrm{~nm}$. Para el grosor de $\mathrm{n}$ capas, multiplicamos el grosor del grafeno por $n$. Es necesario aclarar que es posible emplear otros grosores de la capa de óxido de silicio (los que se pueden encontrar comercialmente) pues como se presenta en la fig. 1d, el valor del contraste óptico cambia al variar el grosor del óxido de silicio, obteniéndose valores óptimos de grosor en 90 y $275 \mathrm{~nm}$, en la literatura se mencionan como valores óptimos a 90 y $280 \mathrm{~nm}$ (Blake et al., 2007).

Necesitamos conocer cuantitativamente la intensidad de la luz reflejada para determinar el número de capas de grafeno que estamos observando. Una idea inmediata sería, iluminar a la muestra con luz monocromática y medir las distintas intensidades de la luz reflejada. Por el proceso de exfoliación, sólo se pueden obtener muestras de grafeno, o pocas capas de grafeno, de algunas decenas de micras cuadradas en área; realizar experimentos ópticos con muestras muy pequeñas, sin el empleo de equipo sofisticado, resulta muy complicado. Una alternativa es adquirir imágenes de las capas de grafeno, vistas al microscopio, y analizar los colores de la imagen con el sistema de color RGB, tal técnica ya se ha propuesto por otros autores (Abergel et al., 2007; Bruna et al., 2008; Craciun et al., 2009), pero para lo propuesto en este trabajo, no se requiere de iluminar monocromáticamente la muestra. Cabe destacar, que el contraste óptico se ha empleado para determinar el número de capas de otros materiales laminares, como diseleniuro de tántalo (2H-TaSe2) (Hajiyev et al., 2013).

Las imágenes adquiridas en el microscopio óptico se pueden analizar con el modelo de color RGB, para tal modelo, el color (C) de cada pixel es una combinación de R unidades del color primario R, G unidades del primario G, y B unidades del primario $\mathrm{B}$ (red, green y blue, por sus siglas en inglés, respectivamente).

Los términos RGB definen un conjunto particular de colores primarios. Los términos RGB indican las cantidades de los primarios requeridos para combinar el color, y son conocidos como valores de triestímulo. El modelo de color RGB se construyó a partir de la percepción humana del color, para tal modelo se utilizaron primarios monocromáticos de longitudes de onda $435.8 \mathrm{~nm}$ (B), $546.1 \mathrm{~nm}$ (G) y $700 \mathrm{~nm}$ (R), una convención establecida por la Comisión Internacional en Iluminación (CIE, por sus siglas en inglés) (D. Fairchild, 2005). Nosotros asociamos por lo tanto a R, G y B, las longitudes de onda de R G y B. Entonces usaremos $\lambda=546.1 \mathrm{~nm}$ en (4), pues el contraste óptico es mayor para esta longitud de onda, como se ve en la fig. 1c. Se deben emplear los valores de los índices de refracción adecuados del silicio y óxido de silicio a esta longitud de onda.

Asociamos una longitud de onda fija con la finalidad de hacer los cálculos del contraste óptico lo más sencillos posibles. Precisamente nuestros resultados muestran que la aproximación es buena al compararla con la espectroscopia Raman (como se verá más adelante). En Ref. (Ying Wang et al., 2012) se usa un intervalo de longitudes de onda para cada componente del sistema RGB.

Por otro lado, la espectroscopia Raman es una herramienta muy útil para determinar el número de capas de grafeno, en general para determinar estructuras de carbono (Ferrari et al., 2006). Las bandas principales de grafeno G y 2D se localizan en 1580 $\mathrm{cm}^{-1}$ y $2700 \mathrm{~cm}^{-1}$, respectivamente. La banda G está asociada con una doble degeneración de modos fonónicos (simetría $E_{2 g}$ ) en el centro de la zona de Brillouin (Tuinstra et al., 1970; Malard, et al., 2009). La banda 2D es el sobre-tono de la banda D, la cual se localiza en $1350 \mathrm{~cm}^{-1}$, y está asociada al desorden estructural en el grafeno o a efec- 
tos de borde. Entonces una muestra de grafeno, libre de defectos, no presenta la banda D. La intensidad y la forma de las bandas G y 2D ayudan a determinar el número de capas de grafeno, por ejemplo, para una capa de grafeno la banda 2D es de 3 a 4 veces más intensa que la banda G (Ferrari et al., 2006).

\section{MÉTOdo EXPERIMENTAL}

Obtenemos grafeno y pocas capas de grafeno a través de la exfoliación mecánica de grafito Kish. El proceso de exfoliación se puede observar a través de internet para una mejor comprensión (https Grafeno Graphene (2012)). Al observar la superficie del sustrato en un microscopio óptico con el objetivo de aumento x100 (Matsuzawa modelo MXT30-UL), se pueden encontrar estructuras como la de la fig. 2. En esta figura se muestra una imagen común de grafeno (región delimitada por las líneas rojas punteadas) y varias capas de grafeno (parte derecha de la zona punteada) iluminadas con luz blanca. Las imágenes han sido adquiridas con una cámara digital Celestron 44421. Se aprecian distintos colores, en particular, el color rosa de las orillas corresponde al sustrato. Como el grafeno es muy delgado, al observarlo en el microscopio óptico, debemos esperar tonalidades cercanas al sustrato, rosas en este caso. Estas imágenes son analizadas en un programa que nos pueda dar los valores RGB de cada pixel de la imagen, en nuestro caso usamos el programa Corel Draw Photo Paint X5.

Para cada tonalidad rosa de la imagen tomamos 50 lecturas del número G, sustrato y capas de grafeno; pues para un sustrato de silicio con $306.2 \mathrm{~nm}$ de óxido de silicio, el contraste óptico máximo se encuentra en las longitudes de onda cercanas al verde, ver fig. 1c. Estos 50 datos se promedian y corresponderán a $I_{S}$ e $I_{G}$ en la ecuación (1),

FIGURA 2. a) capas de grafeno sobre un sustrato de silicio con una película de óxido de silicio con grosor de $306.2 \mathrm{~nm}$, visto a través de un microscopio óptico con aumentos de objetivo X100. En b) se delimita con líneas rojas punteadas la zona que corresponde a grafeno, las zonas de la derecha corresponde a varias capas de grafeno.

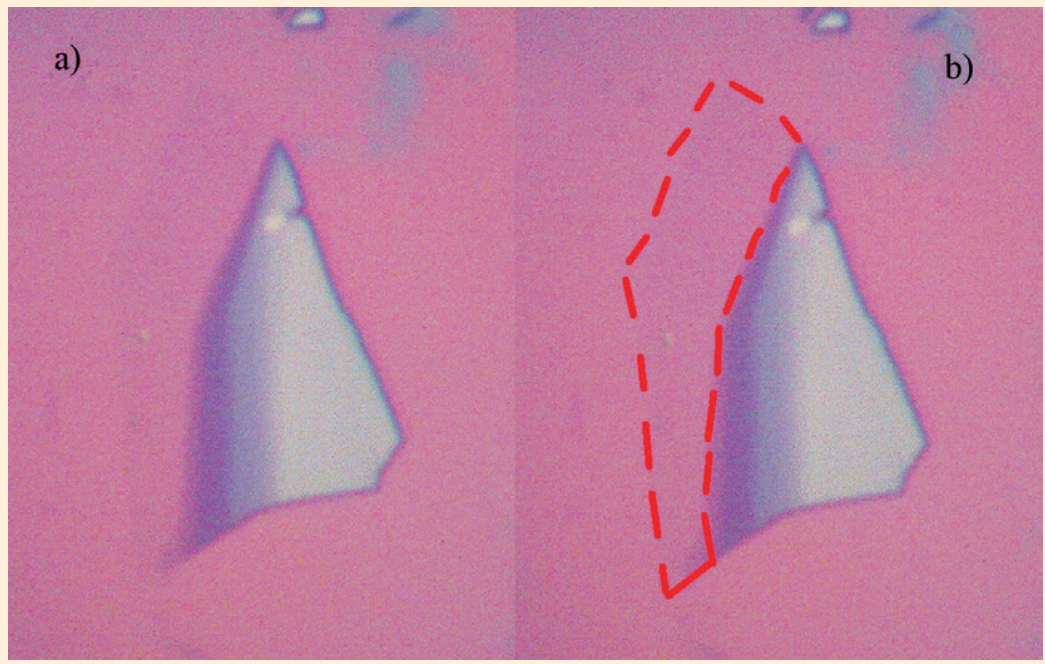


la intensidad de la luz reflejada por el sustrato y las capas de grafeno respectivamente. Se ha sugerido el empleo del programa GIMP para seleccionar de manera libre una determinada zona en las imágenes y obtener el valor promedio de cada color RGB, esto facilita y simplifica de manera considerable la cuantificación del contraste óptico. ${ }^{4}$

El contraste correspondiente al número R también es alto, nosotros sólo hemos usado la componente G; podría también definirse al contraste óptico como una combinación de R y G como en la referencia (Craciun et al., 2009).

Ponemos marcas con una punta de diamante empleando un indentador (Matsuzawa modelo MXT30-UL) alrededor de las muestras de grafeno para hallarlas con mayor facilidad en el microscopio del equipo Raman. Se toman espectros Raman, de las mismas muestras que se han usado para el contraste óptico, con un láser de $532 \mathrm{~nm}$ (NICOLET Almega XR Raman Dispersivo). Analizamos la forma e intensidad de las bandas G y 2D para determinar el número de capas de grafeno a las que corresponde cada espectro, un análisis muy completo de la espectroscopia Raman de grafeno se puede consultar en la referencia (Malard et al., 2009). Posteriormente comparamos el resultado con el contraste óptico.

\section{Resultados}

Como se ha mencionado, la espectroscopia Raman es indispensable para validar los resultados del contraste óptico, es la prueba definitiva en la determinación del número de capas de grafeno. En la fig. 3a se muestran espectros Raman de 1 a 4 capas de grafeno. A medida que el número de capas aumenta, la intensidad de las bandas principales G y 2D varía. La banda G se intensifica, mientras que la banda 2D se ensancha, y se recorre hacia mayores frecuencias.

Para el grafeno, la banda 2D se puede ajustar a una sola lorentziana, mientras que para la bicapa de grafeno es necesario ajustarla a 4 lorentzianas. Para 3 capas de grafeno la banda 2D se ajusta con 6 lorentzianas, y para 4 capas con 3 lorentzianas se reproduce adecuadamente (Malard et al., 2009). De esta manera, podemos identificar con espectroscopia Raman el número de capas de grafeno a las que corresponde cada espectro. Usamos el cociente de las intensidades de las bandas G y 2D para determinar el número de capas a las que corresponde un espectro Raman (Ferrari et al., 2006; Gupta et al., 2006).

En la fig. 3a también se puede apreciar que la banda G casi no se modifica al variar el número de capas de grafeno, mientras que sí existe un claro corrimiento de la banda 2D (Ferrari, 2007). Esto hace notar que la posición de la banda 2D también se puede usar para determinar el número de capas de grafeno. En la fig. 4a se grafica la posición de la banda $\mathrm{G}$ de cada muestra, los primeros 17 datos corresponden a grafeno y los siguientes 20 a bicapa de grafeno. En promedio, para grafeno la banda G se ubica en $1583.5 \mathrm{~cm}^{-1}$, y para la bicapa de grafeno en $1582.12 \mathrm{~cm}^{-1}$. En la fig. $4 \mathrm{~b}$ se presentan las posiciones de la banda 2D. Para grafeno esta banda, en promedio, aparece en $2673.68 \mathrm{~cm}^{-1}$, mientras que para la bicapa de grafeno en $2696.20 \mathrm{~cm}^{-1}$. La diferencia en la posición de esta banda entre grafeno y bicapa es de $22.52 \mathrm{~cm}^{-1}$, en nuestro caso.

\footnotetext{
4 Sugerido por Agustín Torres Oviedo, estudiante del posgrado en ciencias físicas, UNAM.
} 
FIGURA 3. a) Espectros Raman de las capas de grafeno. Ajustes de la banda 2D para b) grafeno, c) 2 capas, d) 3 capas y, e) 4 capas de grafeno.
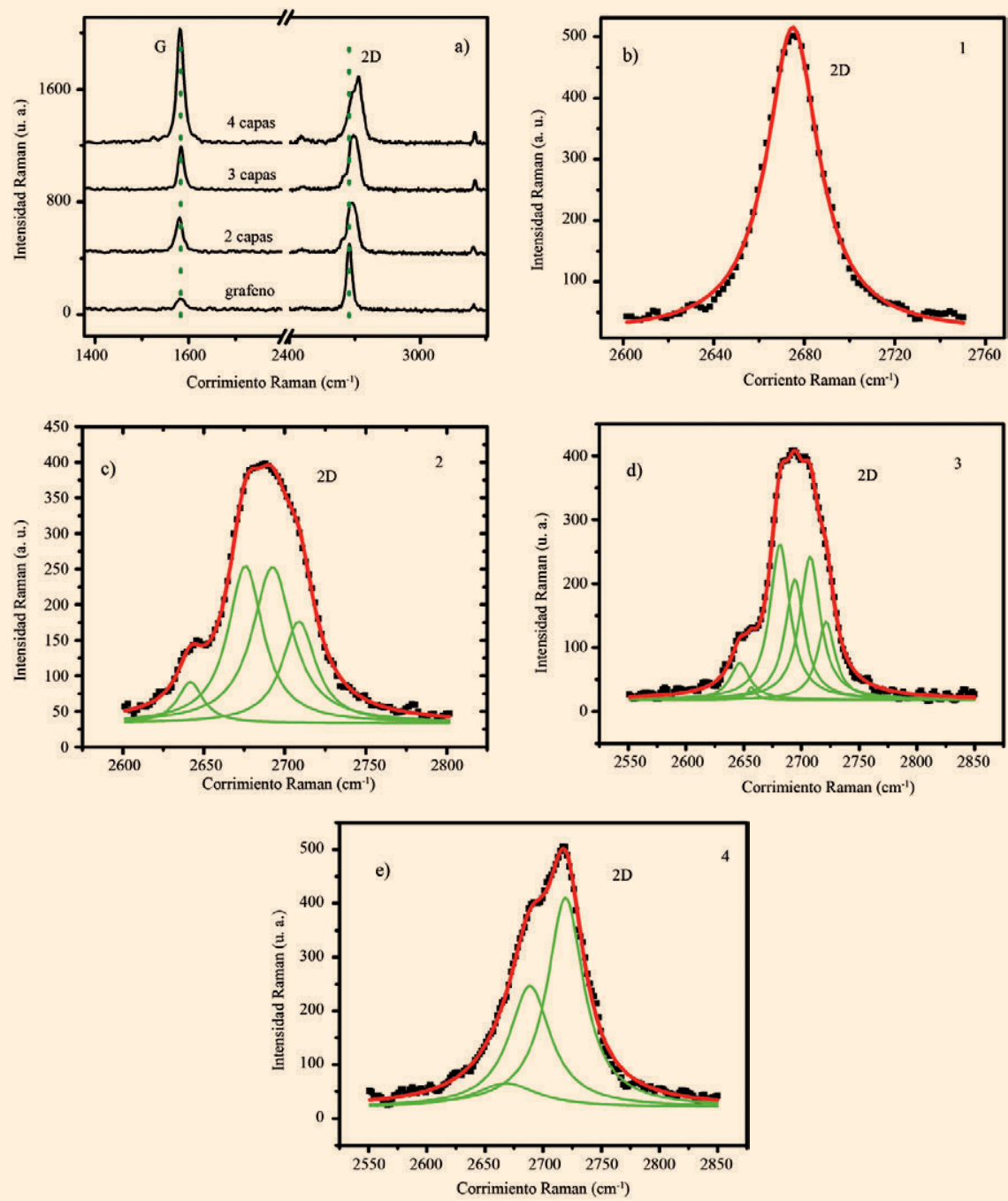

Una vez analizado el espectro Raman de las muestras, comparamos el contraste óptico contra el cociente de las intensidades Raman de las bandas G y 2D y con la posición de la banda 2D. Estos resultados se resumen en la fig. 5. Ambas graficas muestran una correlación positiva entre el contraste óptico y la información del espectro Raman. Tomamos un promedio del contraste óptico de las muestras que con espectroscopia Raman resultaron ser grafeno y bicapa de grafeno. El contraste óptico promedio con su correspondiente desviación estándar es $3.9 \pm 1.06$ y $6.01 \pm 0.901$ para el grafeno y la bicapa de grafeno, respectivamente, mientras que los valores teóricos son 3.57 y 6.52 , respectivamente. 
FIGURA 4. a) Posición de la banda G para grafeno (primeras 17 muestras) y bicapa de grafeno; la posición de la banda $\mathrm{G}$ casi no varía con el número de capas de grafeno. b) Variación de la posición de la banda 2D para grafeno y bicapa de grafeno; la banda 2D se modifica sustancialmente con el número de capas de grafeno.
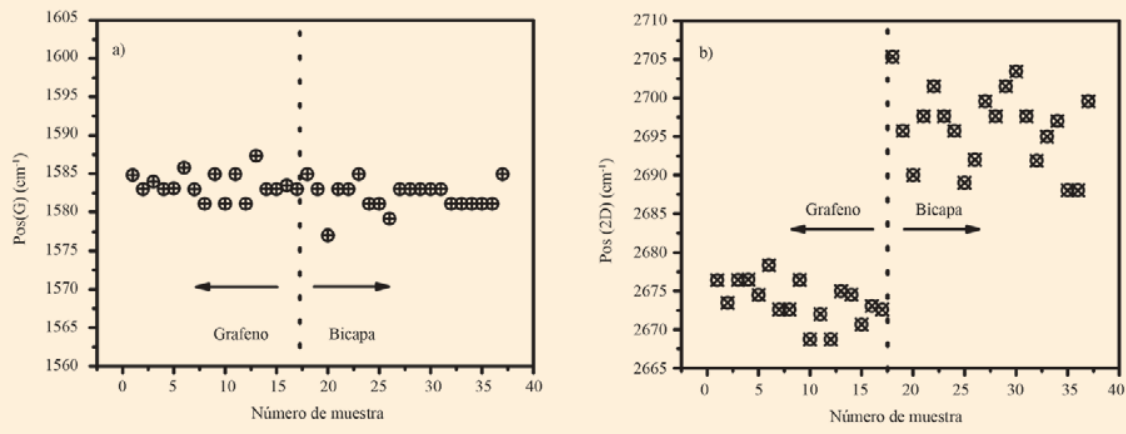

Existen dos observaciones importantes del análisis de los resultados presentados en la fig. 5. Primera, que de la espectroscopia Raman (intensidades relativas de las bandas G, 2D y posición de la banda 2D) se nota claramente que los datos se agrupan para identificar una y dos capas de grafeno; por lo tanto la espectroscopia Raman define de manera inequívoca el número de capas de grafeno. La segunda observación es que el valor del contraste teórico cae dentro de la banda de la desviación estándar de los datos medidos experimentalmente y corroborados con la espectroscopia Raman; esto da un alto grado de confiabilidad de la técnica del contraste óptico.

En nuestras experiencia en el laboratorio, en la pre identificación, establecemos entonces que mientras el contraste óptico se encuentre entre 2.8 y $5 \%$ aseguramos que se trata de grafeno.

La técnica del contraste óptico permite darnos una idea aproximada del número de capas de grafeno, cabe aclarar que es necesario corroborar con la espectroscopia Raman en caso de requerir muestras de grafeno para otro tipo de estudios.

FIGURA 5: a) Contraste óptico contra cociente de intensidades Raman de las bandas G y 2D. b) Contraste óptico contra posición de la banda 2D.
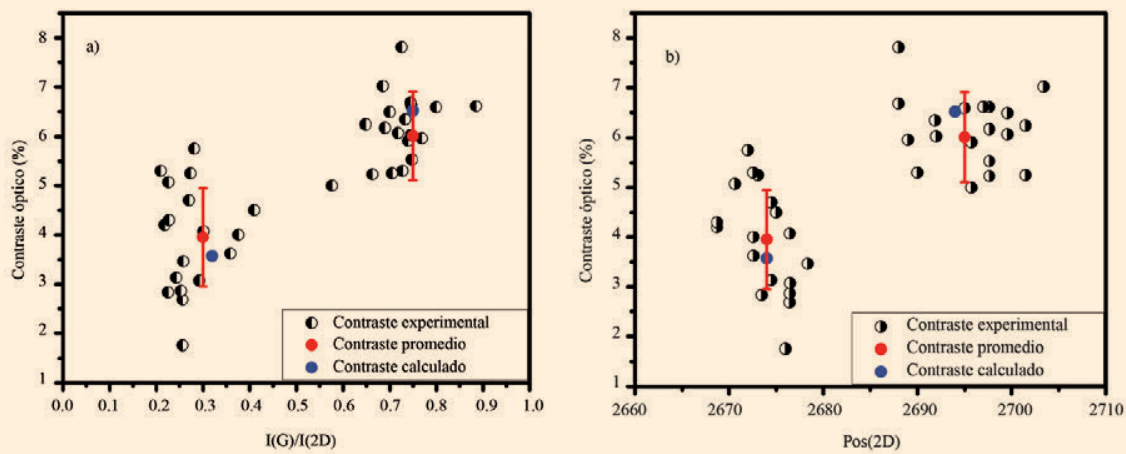
Una vez probado con espectroscopia Raman la confiabilidad del contraste óptico en la determinación del número de capas de grafeno, evaluamos muchas más muestras como se observa en la fig. 6. Las líneas horizontales corresponden al contraste óptico teórico. Se muestra que es posible identificar de 1 a 4 capas de grafeno usando la componente verde del número RGB. Los valores del contraste óptico que se alejan del valor teórico pueden surgir por 2 razones: 1) por residuos de la cinta adhesiva usada en la exfoliación mecánica de grafito o 2) por mala adherencia de las capas del grafeno en el sustrato.

FIGURA 6. Contraste óptico, las líneas horizontales muestran el valor teórico.

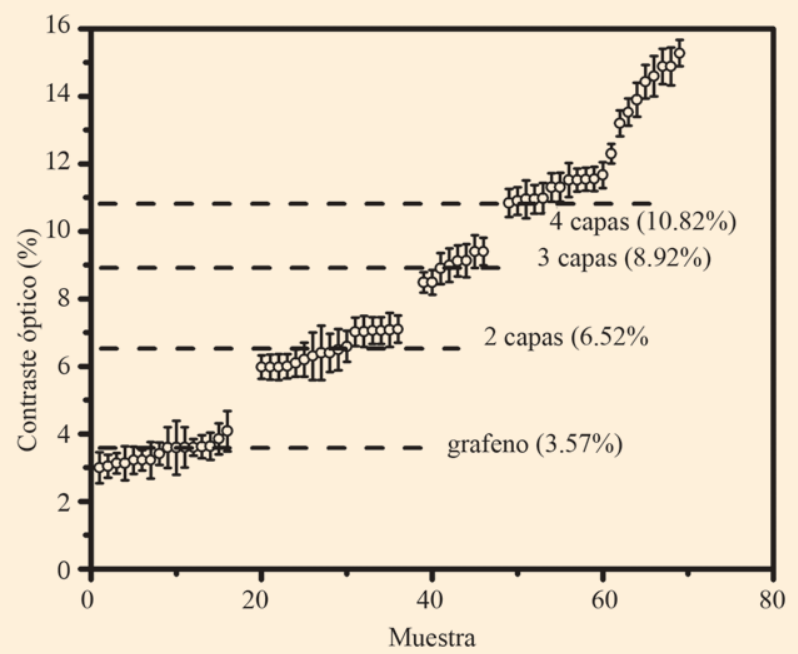

Sugerimos además tener cuidado con: 1) la iluminación en el microscopio óptico, esto es, tratar de usar siempre la misma intensidad; 2) el enfoque de las muestras en el microscopio, 3) defectos de la óptica del microscopio empleado (aberraciones cromáticas, por ejemplo). Cabe aclarar que será necesario emplear espectroscopia Raman para calibrar el método del contraste óptico tomando en cuenta las condiciones específicas de cada laboratorio; por ejemplo, el grosor del óxido de silicio, la óptica del microscopio y la cámara empleada. Pero una vez hecha la comparación entre ambas técnicas, la del contraste óptico expuesta en el presente trabajo se puede emplear como una técnica rutinaria de preidentificación del grafeno.

\section{ConCLUSIÓN}

La espectroscopia Raman es la herramienta ideal en la determinación del número de capas de grafeno. El método del contraste óptico propuesto en este trabajo resulta adecuado para hacer una determinación inicial del número de capas de grafeno, de una a cuatro capas. Por otro lado, la espectroscopia Raman es la herramienta que respalda los resultados obtenidos por el contraste óptico, mostrando que éste último es 
un método aproximado en el cálculo del número de capas de grafeno. Consideramos entonces que es primordial que cualquiera que desee introducirse en el campo de estudio del grafeno, debe necesariamente usar un método visual de determinación del número de capas de grafeno. A diferencia de las propuestas del contraste óptico de otros autores, el método aquí presentado es sencillo y apropiado para realizarse como una práctica de laboratorio a nivel licenciatura en física y en carreras afines.

\section{REFERENCIAS BIBLIOGRÁFICAS}

Abergel, D S. L. (2007) "Visibility of graphene flakes on a dielectric substrate". Applied Physics Letters, vol. 91, 063125.

Anders, H. (1972) Thin films optics. London: Focal Press,.

Alexeev, E. et al. (2013) "Photo-induced doping and strain in exfoliated graphene". Applied Physics Letters, vol. 103, 151907.

Benitez, J. L. y Mendoza, D. (2013) “Modulation of the optical transmittance in multilayer graphene by an electrical signal". Applied Physics Letters, vol. 103, 083116.

Blake, P. et al. (2008) “Graphene-based liquid crystal device”. Nano Letters, vol. 8, núm. 6, 1704.

Blake, P. et al. (2007) “Making graphene visible”. Applied Physics Letters, vol. 91, 063124.

Bruna, M. y Borini, S. (2009) "Optical constants of graphene layers in the visible range". Applied Physics Letters, vol. 94, 031901.

Bunch, J. S. et al. (2008) "Electromechanical Resonators from Graphene Sheets". Science, vol. $315,490$.

Craciun, M. F, et al. (2009) "Trilayer graphene is a semimetal with a gate-tuneable band overlap". Nature Nanotechnology, vol. 94, 0311901.

D. Fairchild, M. (2005) Color appearance models. Londres: John WIley \& Sons.

Ferrari, A. C. et al. (2006) "Raman spectrum of graphene and graphene layers". Physical Review Letters, vol. 97, 187401.

Ferrari, A. C. (2007) "Raman spectroscopy of graphene and graphite: Disorder, electronphonon coupling, doping and nonadiabatic effects". Solid State Communications, vol. $143,47$.

Flores, C. B. y Mendoza, D. (2011) "Multilayer graphene synthesized by CVD using liquid hexane as the carbon precursor". World Journal of Condensed Matter Physics, vol. 1, 157.

Flores, C. B. et al. (2012) “Grafeno: un material con potencial para la tecnología electrónica del futuro". Materiales Avanzados, núm. 19, 17.

Geim, A. K. y Novoselov, K. S. (2007) “The rise of graphene”. Nature, vol. 6, 183.

Geim A. K y Kim, P. (2008) “Carbon Wonderland”. Scientific American, vol. 298, 90.

Grafeno Graphene (2012) <https://www.youtube.com/watch?v=h9KvTgidDzg>.

Gupta, A. et al. (2006) "Raman scattering from high-frequency phonons in supported ngraphene layer films”. Nano Letters, vol. 6, núm. 12, 2667.

Hajiyev, P. et al. (2013) "Contrast and Raman spectroscopy study of single- and few-layered charge density wave material: 2H-TaSe2". Scientific Reports, vol. 3, 2593.

Landau, L. D. (1969) Mecánica estadística. Barcelona: Reverté.

Lee, C. et al. (2008) "Measurement of the elastic properties and intrinsic strength of monolayer graphene". Science, vol. 321, 385. 
Lin, Y. M. et al. (2010) “100-GHz Transistors from wafer-scale epitaxial graphene”. Science, vol. 327, 662.

Liu, C. et al. (2010) "Graphene-based supercapacitor with an ultrahigh energy density". Nano Letters, vol. 10, núm. 12, 4863.

Malard, L. M. et al. (2009) "Raman spectroscopy in graphene”. Physics Reports, vol. 473, 51.

Moriyama, S. et al. (2010) "Fabrication of quantum-dot devices in graphene". Science and Technology of Advanced Materials, vol. 11, 054601.

Nair, P. P. et al. (2008) "Fine structure constant defines visual transparency of graphene". Science, vol. 320, 1308.

Novoselov, K. S. et al. (2004) "Electric field effect in atomically thin carbon films". Science, vol. 306, 666.

Novoselov, K. S. et al. (2005) "Two-dimensional gas of massless Dirac fermions in graphene". Nature, vol. 438, 197.

Palik, E. D. (1991) Handbook of optical constants of solids. New York: Academic Press.

Pereira, V. M. et al. (2008) Physical Review B, vol. 77, 115109.

Ponomarenko, L. A. et al. (2008) “Chaotic Dirac billiard in graphene quantum dots". Science, vol. $320,356$.

Schedin, F. et al. (2007) "Detection of individual gas molecules adsorbed on graphene". Nature, vol. 6, 652.

Stander, N. et al. (2009) "Evidence for Klein tunneling in graphene p-n junctions". Physical Review Letters, vol. 102, 026807.

Tuinstra, F. y Koenig, J. L. (1970) “Raman spectrum of graphite”. Journal of Chemical Physics. vol. 53, núm. 3, 1126.

Wang, L. J. et al. (2010) "A graphene quantum dot with a single electron transistor as an integrated charge sensor". Applied Physics Letters, vol. 97, 262113.

Wang, X. et al. (2008) “Transparent, conductive graphene electrodes for dye-sensitized solar cells". Nano Letters, vol. 8, núm. 1, 323.

Yan, J. et al. (2007) "Electric field effect tuning of electron-phonon coupling in graphene". Physical Review Letters, vol. 98, núm. 16, 166802.

Ying Wang, Y. et al. (2012) “Thickness identification of two-dimensional materials by optical imaging". Nanotechnology, vol. 23, 495713.

Yuanbo, Z. et al. (2005) "Experimental observation of the quantum Hall effect and Berry's phase in graphene". Nature, vol. 438, 201. 